\title{
Extra-nodal natural killer/T cell lymphoma, nasal-type, treated by concurrent chemoradiotherapy
}

\author{
NOBUHIKO YOSHIKAWA, TAISUKE INOMATA, TAIJU SHINBO, \\ MASATSUGU TAKAHASHI, YASUO UESUGI and YOSHIHUMI NARUMI \\ Department of Radiology, Osaka Medical College, Osaka 569-8686, Japan
}

Received February 9, 2010; Accepted March 18, 2010

DOI: 10.3892/etm_00000070

\begin{abstract}
The present study reports on a case of extranodal natural killer/T cell lymphoma, nasal-type (ENKL), stage IIEA, in a 50-year-old woman who presented with a white tumor on a refractory ulcer on the gum. Concurrent chemoradiotherapy was administered, and effected a partial response. However, tumor recurrence was observed 5 months after the final diagnosis, and the patient succumbed 1 month after recurrence. Although a definitive treatment for ENKL has yet to be established due to its rarity, radiation therapy (RT) is crucial to therapy, as ENKL is very sensitive to RT. However, treatment with radiation levels above $50 \mathrm{~Gy}$ with an extended RT field are required for a favorable outcome. The development of novel chemotherapy regimens may therefore be useful. Additionally, autologous or allogenic hematopoietic stem-cell transplantation may prove to be a promising approach.
\end{abstract}

\section{Introduction}

Extra-nodal natural killer (NK)/T cell lymphoma, nasal-type (ENKL), is a distinct entity according to the World Health Organization (WHO) classification of lymphoid tissue. Though rare in Western populations, it occurs with greater frequency among Asians, Mexicans and South Americans of American Indian descent. Due to its rarity and the lack of randomized controlled trials, a definitive treatment for ENKL remains to be established. Here, we describe our experience with a 50-year-old female patient who was diagnosed with ENKL stage IIEA and treated by concurrent chemoradiotherapy. Additional treatment options applied in previous studies are also discussed, including other chemotherapy regimens and autologous or allogenic hematopoietic stemcell transplantation.

Correspondence to: Dr Nobuhiko Yoshikawa, Department of Radiology, Osaka Medical College, 2-7 Daigaku-machi, Takatsuki, Osaka 569-8686, Japan

E-mail: rad101@poh.osaka-med.ac.jp

Key words: extra-nodal NK/T cell lymphoma, nasal-type, concurrent chemoradiotherapy, DeVIC regimen

\section{Case report}

A 50-year-old woman presented at the Osaka Medical College Hospital in April 2008 with a suspected malignant white tumor on a refractory ulcer on the right-lower gum, accompanied by bilateral neck lymph node swelling. Levels of soluble IL-2 receptor $(716 \mathrm{U} / \mathrm{ml})$ were increased. Genetic examination revealed no rearrangement of T-cell receptors or immunoglobulin genes, and no chromosomal abnormalities. CT images revealed a slightly enhanced soft tissue mass with adjacent bone destruction and bilateral neck lymphadenopathy (Fig. 1). No other involved lesions were detected by garium-67 scintigraphy. Histological examination of biopsy specimens with $\mathrm{H} \& \mathrm{E}$ staining revealed diffuse infiltrate lymphoma cells. The immunophenotype was $\mathrm{CD}_{56}{ }^{+}$, cytoplasmic $\mathrm{CD}^{+/-}, \mathrm{CD}^{+} 6^{+}$, granzyme $\mathrm{B}^{+}, \mathrm{CD}^{2} 0^{-}, \mathrm{CD}^{-}$- and $\mathrm{CD} 8$ - The specimens were also positive for Epstein-Barr virus encoded RNA (EBER) (Fig. 2).

The tumor was diagnosed as ENKL stage IIEA. Concurrent chemoradiotherapy was administered according to the regimen described in the Japan Clinical Oncology Group (JCOG) trial 0211-DI (Fig. 3) (1). This protocol was recommended since ENKL is intractable and lacks a standard therapy. However, the case was not registered with the JCOG trial, and the protocol was not approved by an institutional review board, since it was necessary to commence treatment immediately. The patient provided informed written consent.

All extra-nodal lesions identified in the CT images as well as the bilateral neck and supraclavicular lymph node regions were included within the radiation portals. Using a 4-MV photon beam, $41.4 \mathrm{~Gy}$ in 23 fractions over 4.6 weeks was delivered to the extra-nodal site and to the neck and supraclavicular lymph node regions (Fig. 4A). Subsequently, the radiation portals were modified to avoid the spinal cord, and 9.0 Gy in 5 fractions over a week was delivered to the lesions with 4- and 10-MV photon beams (Fig. 4B). A 10-MeV electron beam was employed to adequately cover the mandibular tumor area. The patient suffered from grade 3 neutropenia and grade 3 acute oral mucositis, which required temporary intravenous hyperalimentation.

On CT images captured 7 days after the completion of the radiation therapy, the therapeutic effect was determined to be a partial response. Five months after the final diagnosis, both in-field and distant tumor recurrences were observed on PET/CT 
A

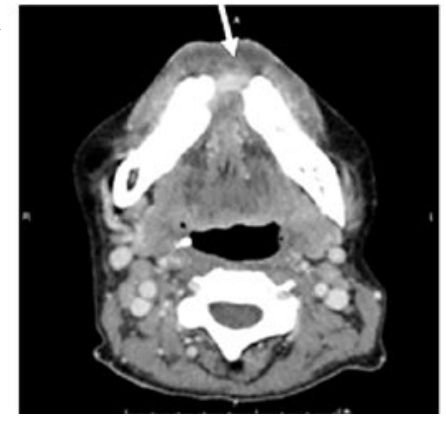

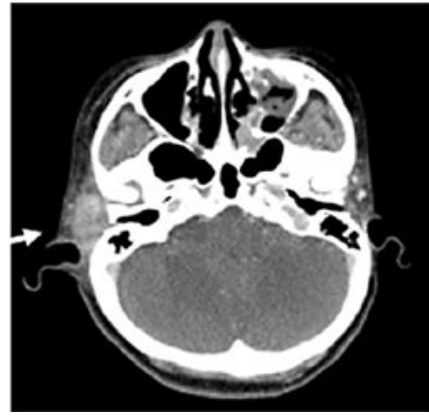

C

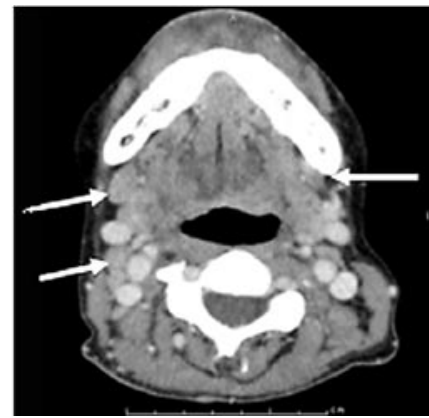

Figure 1. CT images of ENKL prior to the initial treatment. (A) Soft tissue mass adjacent to the mandible. (B) Soft tissue mass with slight contrast enhancement at the right temporal lobe. (C) Bilateral neck lymphadenopathy.

A

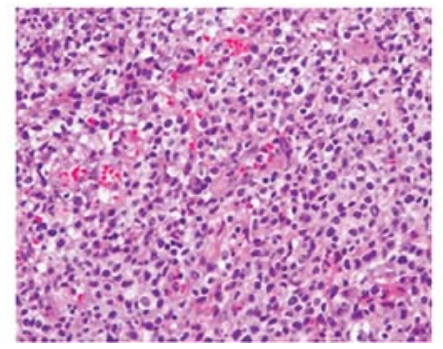

B

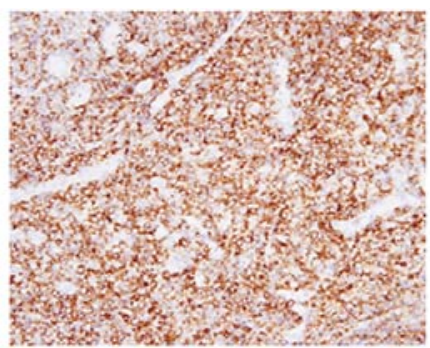

C

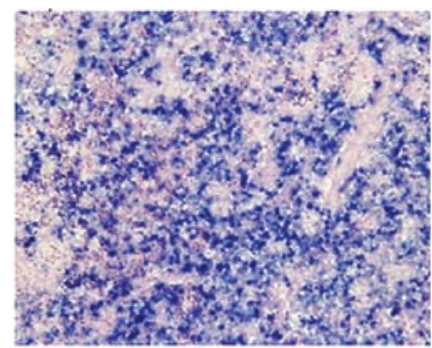

Figure 2. Biopsy specimens from the gum. (A) H\&E staining of a specimen showing diffuse infiltrate lymphoma cells. (B) Specimen positive for CD56 determined using an enzyme antibody technique. (C) Specimen positive for Epstein-Barr virus encoded RNA determined by in situ hybridyzation.

2/3DeVIC:

CBDCA $200 \mathrm{mg} / \mathrm{m}^{2}$ day 1, ETP $67 \mathrm{mg} / \mathrm{m}^{2}$ day $1-3$

IFM $1.0 \mathrm{~g} / \mathrm{m}^{2}$ day $1-3$, Dex $40 \mathrm{mg} /$ body day $1-3$

Day 1

2/3DeVIC

(1) course

Day 30

Day 72

$\downarrow$

(2) course

(3) course
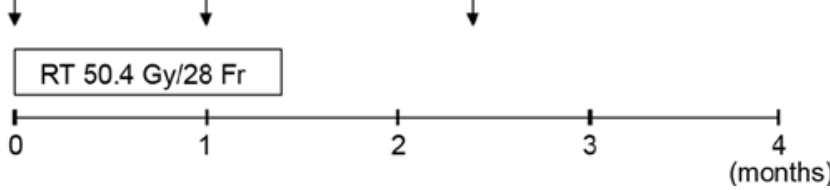

Figure 3. Schedule and dosage of concurrent chemoradiotherapy (RT-2/3DeVIC regimen, Japan Clinical Oncology Group trial 0211-DI).

images. Salvage chemotherapy (JALSG ALL202 protocol) was performed, but the patient succumbed 1 month after recurrence.

\section{Discussion}

ENKL is characterized histologically by angiocentric and polymorphous lymphoreticular infiltrates, and clinically by progressive necrotic lesions occurring mainly in the nasal cavity and a poor prognosis caused by rapid progression of the lesion into distinct organs. This lymphoma is very rare in Western populations, but occurs with greater frequency among Asians, Mexicans and South Americans of American Indian descent. It has been reported that NK/T cell lymphoma accounts for approximately $2.6 \%$ of all malignant lymphomas in Japan (2). ENKL is subcategorized into 'nasal' and 'nasal-type' ENKL according to the anatomic sites of the primary disease. In previous reports, nasal ENKL has
A

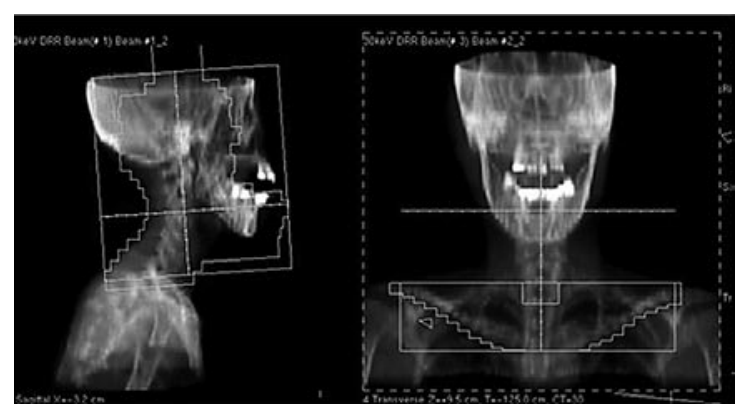

B

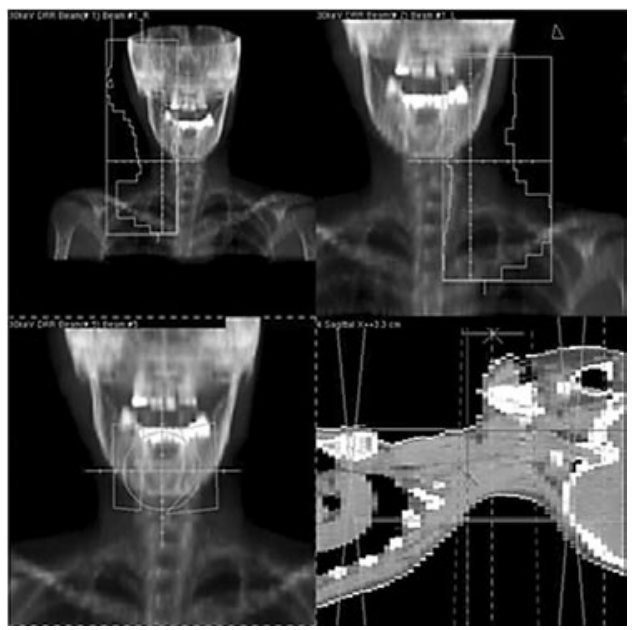

Figure 4. Arrangement of the irradiated field. (A) From 0 to $41.4 \mathrm{~Gy}$, two lateral opposed portals to the head and neck tumor and an anterior beam to the supraclavicular lymph node area were employed with a 4-MV photon beam. (B) From 41.4 to $50.4 \mathrm{~Gy}$, anterior-posterior portals with 4- and 10-MV photon beams were employed to avoid the spinal cord, and the mandibular tumor area was irradiated with a $10-\mathrm{MeV}$ electron beam. 
been observed in the nasal cavity and upper aerodigestive area, while nasal-type ENKL has been noted in other sites $(4,5)$. The nose and paranasal area, including the upper aerodigestive tract, is the origin of more than $80 \%$ of ENKL occurences. The skin is the second most frequent organ of origin, accounting for approximately $10 \%$ of occurrences, followed by the liver and/or spleen at $5 \%$ and, more rarely, the lungs, gastrointestinal tract, kidney, pancreas, testis and brain (7). Clinical characteristics differ between nasal and nasal-type ENKL: nasal-type ENKL is more advanced at diagnosis, and therefore, has a poorer prognosis than nasal ENKL (4).

As ENKL is very sensitive to radiation therapy (RT), RT plays a crucial role in its treatment, especially during the limited stages of the disease (stages I/II). Previous reports have suggested that RT as an initial treatment improves the local control probability (LCP) and overall survival (OS) of patients (5). Isobe et al reported that patients who received over 50 Gy with an RT field that included potential contiguous areas (the paranasal sinuses, palate and nasopharynx) with an adequate margin had a higher LCP. These results were statistically significant (5). Koom et al analyzed the doseresponse relationships for local control, which showed a sigmoidal shape and reached a plateau at doses in excess of 54 Gy. However, the authors also found that dose escalation in patients who do not attain a complete response after the planned RT dose may improve the LCP (9). Therefore, doses over $50 \mathrm{~Gy}$ and an extended RT field are considered to confer a favorable prognosis (8).

Currently, combined chemotherapy is performed in many cases of ENKL, (3-7,9). However, ENKL is often resistant to the anthracycline-based chemotherapy used for malignant lymphomas $(3,7)$. One possible explanation for this resistance is that ENKL commonly expresses P-glycoprotein, which is a product of the multidrug resistance-1 gene (11) and has an effect on anthracycline. Several reports have concluded that the addition of current chemotherapy regimes to radiation therapy for the treatment of ENKL may not improve the OS of the patients $(4,6)$. Therefore, the development of effective chemotherapy regimens is an important step towards improving the prognosis of both limited and advanced stage ENKL. Several clinical studies of potential regimens are ongoing. For limited stage ENKL, Yamaguchi et al reported a favorable treatment outcome with concurrent chemoradiotherapy according to a DeVIC regimen (3), which consists of carboplatin, ifosphamide, etoposide and dexamethasone. Carboplatin and ifosphamide are not affected by P-glycoprotein, and etoposide is a key drug for the treatment of Epstein-Bar virus-related hemophagocytic syndrome. For advanced stage or relapsed ENKL, SMILE chemotherapy (consisting of L-asparaginase, methotrexate, ifosphamide, etoposide and dexamethasone) (10) and autologous or allogenic hematopoietic stem-cell transplantation have been reported to be effective, though these studies have only been conducted in a small patient sample.
In the current case, a complete response was not achieved after the completion of treatment, and no therapeutic effects of treatment were observed after recurrence. According to previous reports, the survival curves decrease abruptly within 1-2 years of treatment and later increase very slowly (4-9). Patients with a complete response and good local control tend to achieve a longer survival time $(3,9)$. These studies and our current report indicate that additional boost irradiation may improve patient outcome. Furthermore, intensive follow-up and speedy induction salvage chemoradiotherapy should be performed.

\section{References}

1. Yamaguchi M, Tobinai K, Oguchi M, et al: Phase I/II Study of Concurrent Chemoradiotherapy for Localized Nasal Natural Killer/T-Cell Lymphoma: Japan Clinical Oncology Group Study JCOG0211. J Clin Oncol 27: 5594-6001, 2009.

2. Lymphoma Study Group of Japanese Pathologists: The World Health Organization classification of malignant lymphomas in Japan: incidence of recently recognized entities. Pathol Int 50: 696-702, 2000.

3. Yamaguchi M, Ogawa S, Nomoto Y, et al: Treatment outcome of nasal NK-T cell lymphoma: a report of 12 consecutivelydiagnosed cases and a review of the literature. J Clin Exp Hematopathol 41: 93-99, 2001.

4. Cheung MMC, Chan JKC, Lau WH, Ngan RKC and Foo WWL: Early stage nasal T/NK-cell lymphoma: clinical outcome, prognostic factors, and the effect of treatment modality. Int J Radiat Oncol Biol Phys 54: 182-190, 2002.

5. Isobe K, Uno T, Tamaru J, et al: Extranodal natural killer/T-cell lymphoma, nasal type. Cancer 106: 609-615, 2006.

6. Kim K, Chie EK, Kim CW, Kim IH and Park CI: Treatment outcome of angiocentric T-cell and NK/T-cell lymphoma, nasal type: radiotherapy versus chemoradiotherapy. Jpn J Clin Oncol 35: 1-5, 2005.

7. Suzuki R, Takeuchi K, Ohshima K and Nakamura S: Extranodal NK/T-cell lymphoma: diagnosis and treatment cues. Hematol Oncol 26: 66-72, 2008.

8. Shikama N, Ikeda H, Nakamura S, et al: Localized aggressive non-Hodgkin's lymphoma of the nasal cavity: a survey by the Japan Lymphoma Radiation Therapy Group. Int J Radiat Oncol Biol Phys 51: 1228-1233, 2001.

9. Koom W, Chung E, Yang W, et al: Angiocentric T-cell and NK/T-cell lymphomas: radiotherapeutic viewpoints. Int J Radiat Oncol Biol Phys 59: 1127-1137, 2004.

10. Yamaguchi M, Suzuki R, Kwong YL, et al: Phase I study of dexamethasone, methotrexate, ifosfamide, L-asparaginase, and etoposide (SMILE) chemotherapy for advanced-stage, relapsed or refractory extranodal natural killer $(\mathrm{NK}) / \mathrm{T}$-cell lymphoma and leukemia. Cancer Sci 99: 1016-1020, 2008.

11. Egashira $M$, Kawamata $N$, Sugimoto $K$, Kaneko $T$ and Oshimi K: P-glycoprotein expression on normal and abnormally expanded natural killer and inhibition of P-glycoprotein function by cyclosporine A and its analogue, P SC83. Blood 93: 599-606, 1999. 